\title{
Seúl y el Río Han: 1945-2014
}

\section{Seoul and Han River: 1945- 2014}

\section{Eunmi Kim^}

Fecha de superación del Tribunal Fin de Máster: 16.07.2015

Tutor: Julio Pozueta Echavarri

\section{Resumen}

Éste artículo contribuye al entendimiento de la evolución de la ciudad de Seúl en torno al Río Han a través del análisis cruzado de su morfología urbana en relación a la situación política y económica, la estrategia urbana y el papel de los planes urbanísticos, así como de las grandes operaciones urbanísticas, en torno al río Han, tanto en los crecimientos de su orilla sur (Gang-Nam), como la transformación del centro histórico en el norte. El río Han y sus alrededores reflejan el gran cambio de la morfología de Seúl a través de su historia, en la que la relación entre la ciudad de Seúl y el río ha tenido un papel muy importante en la evolución urbana, pasando de ser un área exterior a la zona de influencia de la antigua capital, a convertirse en su centro geográfico con la extensión de la ciudad moderna al sur del río, influenciada por la situación de conflicto existente entre las dos Coreas, y derivando en un gran desequilibrio entre el Norte y el Sur de la ciudad.

\section{Palabras clave}

Seúl, Río Han, evolución, morfología, extensión al sur del río, desequilibrio entre norte y sur del río

\section{Abstract}

This article contributes to the understanding of the evolution of Seoul city around the Han River through cross analysis of their urban morphology in relation to the political and economic situation, the urban strategy and the role of urban planning, as well as the great urban operations around the Han River, in the growth of its southern shore (Gang-Nam) and the transformation of the historic center in the north. The Han River and its surroundings reflect the great change in the morphology of Seoul through its history, in which the relationship between the city of Seoul and the river has played an important role in urban development, from being an area outside the area of influence of the former capital, to a geographical center to the extension of the modern city south of the river, influenced by the situation of conflict between the two Koreas, and resulting in a disequilibrium between the North and south of the city.

\section{Keywords}

Seoul, Han River, evolution, morphology, extension of the south of the river, disequilibrium between the North and south of the city

\section{Introducción}

La relación de la ciudad con su río ha tenido un papel muy importante en la evolución urbana de Seúl. El río Han se encontraba fuera del centro urbano en la antigüedad hasta que, con la extensión de la ciudad hacia el sur, se convirtió en su centro geográfico. Este hecho deriva en un primer momento de la decisión de repartir los distintos focos de desarrollo urbano por el territorio,

\footnotetext{
- Eunmi Kim es alumno/a de postgrado del Departamento de Urbanística y Ordenación del Territorio de la Escuela Superior de Arquitectura. Universidad Politécnica de Madrid, mail : eunmik90@gmail.com
} 
como estrategia de tipo defensivo a raíz del conflicto entre Corea del Norte y Sur, ocupando las zonas más accesibles en la llanura del Han.

En la primera mitad de siglo XX, Seúl atravesó momentos muy convulsos de su historia, con un período de colonización entre 1909 y 1945, seguido de la guerra de tres años que terminaría en 1953, dividiendo los dos países. Tras la guerra, la ciudad entró en un proceso de crecimiento muy acelerado, con una población que pasaría de 1.5 millones de habitantes en aquel momento, a alcanzar los 10 millones de habitantes en 1990, cifra que se ha mantenido relativamente constante hasta la actualidad.

Durante el período de conflicto el país se organizó bajo gobiernos militares ante la constante amenaza de hostilidad bélica. La preocupación por la seguridad de los ciudadanos animó al gobierno local a promover la extensión de la ciudad como estrategia de dispersión consciente de los usos urbanos. En 1963, se decidió ampliar el área metropolitana de Seúl hacia al sur, convirtiendo la llanura del Río Han en su mayor eje de desarrollo, y motivando una gran inversión en infraestructuras de contención y transporte, con la construcción de diques, autopistas paralelas al río y gran cantidad de puentes, que transformarían por completo la geografía ancestral del río como eje de transporte y reserva natural.

Para la extensión y el desarrollo del sur de río, las autoridades de planificación urbana propusieron una estructura espacial organizada en múltiples sub-centros repartidos por la región, que evolucionaría a una estrategia más simple centrada en tres núcleos: El distrito del Redesarrollo urbano (CBD) en el centro histórico, el Distrito Yeongdeungpo en el sur-oeste del río y Distrito Gangnam en el sur-este del río, a través de políticas de traslado de los Edificios Públicos y nuevas áreas de concentración empresarial, con zonas asociadas de alta densidad residencial. Con el paso del tiempo, estos núcleos fueron experimentando un crecimiento muy desigual. Mientras que el sur-este del río se desarrolló enormemente, el centro histórico únicamente experimentó apenas un pequeño aumento en el distrito de Re-desarrollo urbano, (con una política firme de contención de la urbanización en el norte del Han) y el núcleo sur-oeste mantuvo el carácter principalmente industrial heredado de la época colonial. Éste proceso muestra haber configurado el nuevo Seúl con un fuerte desequilibrio y segregación espacial entre norte y sur, este y oeste evidenciado por la distribución actual de usos, morfologías urbanas, y características de su población.

Después del cambio del régimen militar por el gobierno democrático (1993) y el comienzo de la autonomía local, Seúl transformó su modelo urbano hacia el desarrollo sostenible, la cohesión y el equilibrio entre las localidades, recuperando gran cantidad de elementos históricos y naturales, como el área de Cheonggyecheon en el centro histórico, la rehabilitación ecológica de las islas del río Han, Tukseom, Seonyudo y Nanjido, que se convirtieron en grandes parques urbanos. En la actualidad, se está promoviendo el desarrollo hacia al Oeste y la recuperación del tejido urbano en el centro histórico, que coexiste con el aún intenso desarrollo de los núcleos urbanos del sur del río.

Muchas grandes operaciones urbanísticas se desarrollaron en torno del Río Han, por las que se puede leer la morfología urbana de Seúl. También los proyectos de regeneración urbana como curación extrema de la última etapa reflejan que cómo se acumularon los problemas a largo de la última mitad del siglo XX y, cómo influyen las estrategias urbanas y los planes urbanos al desarrollo de Seúl.

\subsection{Objetivos de la investigación}

El objeto de este estudio es entender las causas de la evolución urbana de Seúl de un modelo físico compacto a una estructura dispersa y segregada por el río Han, mediante el análisis del cambio de su morfología en relación con la situación política y económica, así como con la estrategia urbana y el papel del planeamiento en el proceso. Además, se van a revisar grandes operaciones urbanísticas en torno al Río Han, y la transformación del centro histórico en el norte con el ánimo de ejemplificar mejor el proceso evolutivo de Seúl. 


\subsection{La metodología}

Estudio de las principales etapas de la evolución urbana de Seúl, en torno del río

- Etapa previa: hasta 1960.

- Etapa I (1961-1979):

la fase del inicio del desarrollo urbano

- Etapa II (1980-1992):

la fase del desarrollo

- Etapa III (hasta la actualidad):

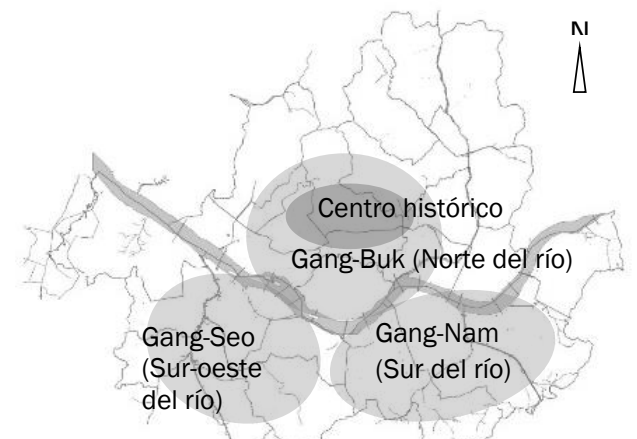

la fase de Regeneración urbana

Materiales empleados: estrategias urbanas, planes, prensa, planos, documentos, fotografías, mapas.

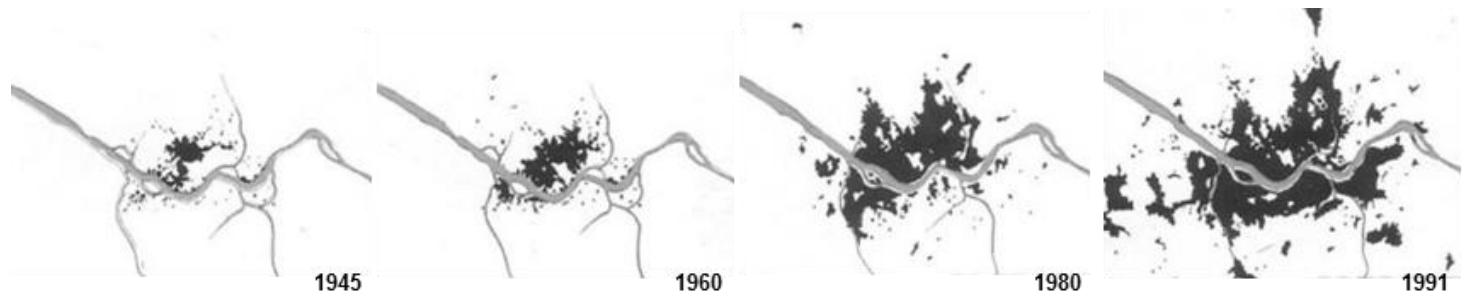

Figura 1. Evolución urbana de Seúl. Fuente: http://ehistory.go.kr , 'Capital Seúl, 600 años’, 1994

\section{Etapa Previa (Hasta 1960)}

\subsection{Breve historia de Seúl. Principales condicionantes ancestrales y desarrollo hasta el fin de la guerra.}

La antigua Seúl fue fundada en 1394, y su establecimiento se basó en la filosofía del emplazamiento que consideraba los atributos geográficos de la tierra desde la idea del confucianismo y la teoría Feng-Shui. La ciudad creció en torno a un esquema que ubicaba el palacio real y su alcázar en el centro, y desde el que partían las direcciones cardinales, que iban adaptando su trama urbana a las 8 montañas que lo rodeaban (cuatro dentro del límite fortificado y cuatro fuera de él), y su arroyo principal (Cheonggyecheon), con un recinto amurallado de 4 puertas (Sadaemoon). Separada del río Han en origen por una cadena montañosa, su principal eje de desarrollo estaba dirigido hacia al sur-oeste donde se ubicaba el principal puerto, Incheon. Con la construcción del Puente Hangang y el puente del ferrocarril hasta el puerto durante la colonización japonesa (1909-1945), se desarrolló la zona industrial de Yeongdeungpo (sur-oeste del río) y la zona Seongsu en el este. En etapas posteriores se irían desarrollando distintas islas en el río, y se ocuparía la extensa zona de la base militar de EEUU. 


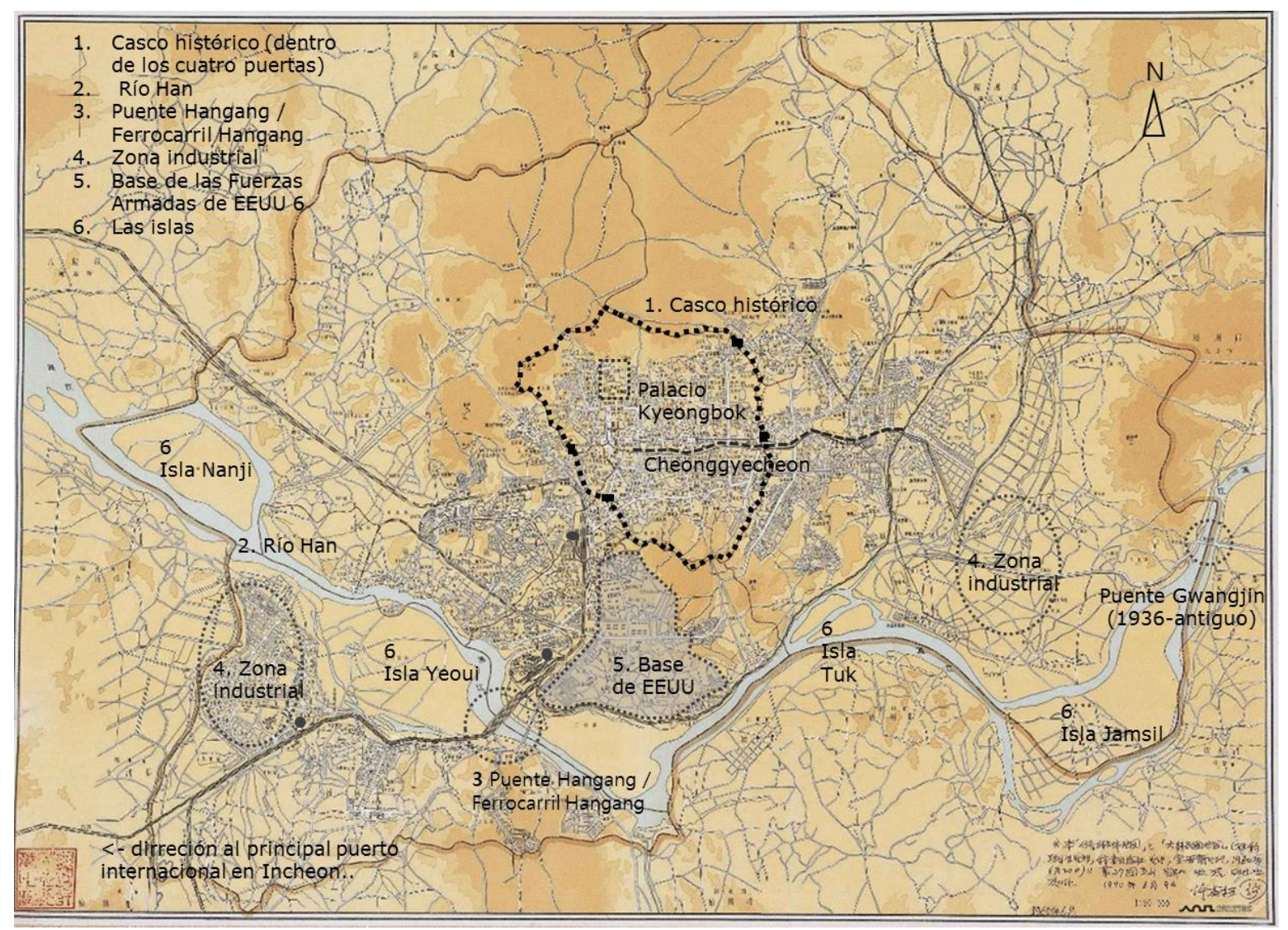

Figura 2. Seoul teukbyeolsi jido, Mapa de Seúl,1960 Fuente: Museo de la Historia de Seúl

Tras el auge del movimiento comunista en el norte de Corea, la guerra estalló en 1950. Tras 3 años de guerra, Corea se dividió en dos países con el armisticio de 1953, entrando en dos sucesivos períodos de gobierno republicanos con una política dominada por la ideología anticomunista.

En el área reconstruida tras la guerra, la población de Seúl experimentó una intensa inmigración rural, y pasó de contar con 1,4 millones de habitantes en 1949, a 2,4 millones en 1960. Éste proceso incrementó la vulnerabilidad estratégica (en términos defensivos) de todo el área urbana, motivando políticas de re-ordenación del suelo y ampliación de las infraestructuras principales, etc. sin un plan urbano definido a largo plazo.

\section{Etapa I (1961-1979)}

El cambio de la morfología de Seúl comenzó a hacerse más evidente en la época de los años 60 a los 70. El área de Metropolitana se extendió hasta el Sur del Río Han, que quedaría ubicado en el centro del acelerado desarrollo de la ciudad moderna.

\subsection{Principales acontecimientos en el contexto urbano de Seúl.}

El golpe militar en 1960 daría comienzo del largo período de dictadura militar hasta el fallecimiento del dictador en 1979. Las políticas nacionales estaban centradas principalmente en el desarrollo económico y la política anticomunista contra Corea del Norte como motivación de toda su agenda. Diversos planes quinquenales se fijaban como plazos y objetivos de desarrollo a nivel nacional, con la sombra del ataque de la vecina septentrional como acicate para el refuerzo de la diezmada economía del país. 
La división de las dos Coreas, y la ubicación relativa de Seúl, muy próxima a la frontera, motivó la extensión de la ciudad hacia el sur, lejos de la misma, y la adopción de políticas anticomunistas y defensivas en su gestión y desarrollo. Se acometieron y justificaron los túneles urbanos como foso de defensa, la red de carreteras como elemento de vigilancia del territorio, las construcciones de carácter simbólico anti comunista en las 4 montañas principales de Seúl ${ }^{1}$, y un gran número de equipamientos relacionados con instalaciones de defensa nacional que ocuparon los suelos de mayor accesibilidad, como es el caso de la Base de EEUU en Yongsan, y sus zonas residenciales asociadas.

Por otro lado, la población de Seúl, que era de 2,4 millones en 1960, se incrementó a 5.4 millones en 1970 y alcanzó los 8.4 millones en 1980. El gobierno local, dentro de su línea de acción, propuso una estrategia de dispersión de la población, dada la preocupación por la seguridad de los ciudadanos ante eventuales ataques del Norte, comenzando la migración del desarrollo urbano hacia el Sur del río. En esta etapa, como se ve, observamos las primeras evidencias de la ruptura de una cierta armonía entre el contexto urbano y su territorio.

\subsection{Estrategias urbanas estatales, y planes urbanísticos metropolitanos}

Para la extensión hacia el sur, en 1963 se aumenta la superficie administrada como área Metropolitana de Seúl con una superficie de $593.75 \mathrm{~km}^{2}$ (que en la actualidad alcanza ya los 605.18 $\left.\mathrm{km}^{2}\right)$. Para promover esta extensión al sur se plantearon diferentes estrategias urbanas:

- 1 Plan del Desarrollo integral del Río Han (1967-1970)

- Restricción del desarrollo del norte del río (1975-),

- Traslado de los equipamientos públicos al sur del río y más allá de Seúl (1978-)

\section{Planes urbanísticos de etapa I: Anexo-1}

- 1966: First City Master Plan toward 1985,

- 1972: City Comprehensive Master Plan toward 1991,

- 1978: Proposal for Urban Master plan toward 2001

Observamos que en un período de 15 años se proponen una gran variedad de estructuras de desarrollo policéntrico. El primer esbozo para un Plan de Seúl se habría llevado a cabo en 1966, dotado de un centro fuerte con 6 sub-centros ( 3 al norte del río y 3 en la margen sur) como estructura espacial para alcanzar un cierto equilibrio en el desarrollo.

En 1972 se añadieron a esta primera estrategia 2 sub-centros en el sur-oeste, y la propuesta en 1975 del concepto "Samhaek-dosi (Ciudad de 3 Núcleos)" por el urbanista Kim, H,-M., planeó la distribución de la función centrada en el principal CBD por tres para la dispersión de la población. Podría Ver que la idea se generó por el límite del casco histórico rodeado las montañas. E influyó la distribución al traslado de los Equipamientos Públicos, y se promovió el desarrollo centrado en el Distrito Gangnam y la isla Yeoui en Distrito Yeongdeungpo. El plan de 1978 quedaría establecido en una combinación del plan original de sub-centros, con la idea de establecer tres núcleos más fuertes para organizarlos. Ninguno de estos planes llegó a sancionarse por las grandes disputas sobre la ubicación de las áreas de desarrollo, pero son una buena muestra de la actitud de dispersión en la política de crecimiento urbano de Seúl como precedente a su expansión posterior.

\footnotetext{
${ }^{1}$ Algunos de ellos, trasladaron a otras ciudades del sur más allá de Seúl en la epata siguiente, demás ha venido separando lo públicos o privatizando, según cambio en régimen democrática. Y por igual razón, intentó construirse Capital temporal en un provincia del sur (1975), pero se anuló, en 1978, se construyó $2^{\circ}$ Edificio de Gobierno en Gwacheon-Si que está en el sur de Seúl. AHN, C.-M. (2013).
} 


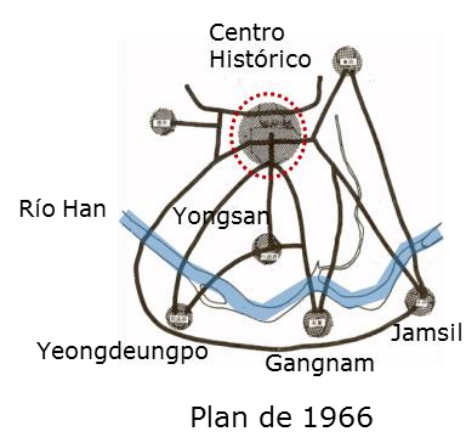

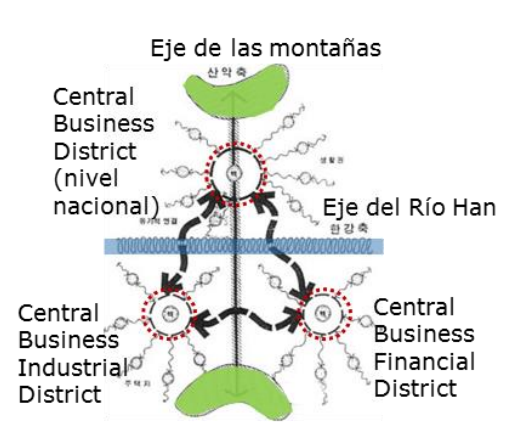

El concepto "Ciudad de 3 Núcleos “, 1975

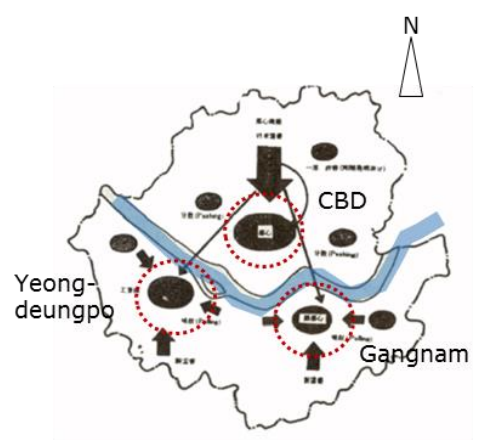

Plan de 1978

Figura 3. Cambio del plan de estructura espacial de Seúl: 1966, 1978 Fuente: Plan de Seúl, 1997, SOHN, J.- M. $2003, n^{\circ} 3$.

\subsection{Grandes operaciones urbanísticas}

En ésta primera fase, la actuación urbana con mayor impacto fue el primer Plan de desarrollo integral del río Han, que inició una serie de obras de infraestructura sobre el mismo, como diques para contener sus periódicas inundaciones, y hasta 9 puentes. Sobre la estructura de diques, se construyó la primera red de autopistas, modificando por completo la topografía del río, con grandes islas del Han desapareciendo por la explotación de gravas y arenas (como la isla Bamseom, que desapareció para la construcción de otra isla, la isla Yeoui, y las islas de Tuk y Jamsil, completamente transformadas por la extracción de tierra.

Dentro del Plan de desarrollo de río Han, la historia de la isla Yeoui es considerada como el paradigma del desarrollo de Seúl, ya que se planeó estratégicamente como centro del sur-oeste del río (Ver R1 en Figura-4). Se aplicó la primera planificación urbana por un arquitecto, pese a un plan imposible de realizar en aquel tiempo ${ }^{2}$. En la actualidad, queda la forma lineal en el suelo. En esta etapa, a la isla, se trasladaron el edificio de la Asamblea Nacional y Asociación Económica, y la sede de KBS (1976 - Corporaciones de difusión) y etc. También se construyó "Yeouido Sibeom Apat (Apartamento piloto) ${ }^{3}$ " como primer edificio de apartamentos en altura, de 15 plantas, vendido a funcionarios y a la gente acomoda en 1970. Influyó a los ciudadanos en la visión y aceptación de la vida en apartamentos, ya que en aquel tiempo, aún no se habían acostumbrado a la vida en apartamentos. Se aceleró la construcción de gran cantidad de apartamentos sobre los suelos del lado del río (Ver R2 en Figura-4).

\footnotetext{
${ }^{2}$ Fue diseñado por el arquitecto Kim, Su-Geun, se propuso la extensión lineal y la ciudad tridimencional con el concepto de megaestructura de Danke Genzo. SOHN, J.- M. 2003, vol 2.

${ }^{3}$ Se eqipó el sistema central de calefacción y aceso, se ha mantenido como emblema de riqueza hasta la actualidad. SOHN, J.- M. 2003, vol 2.
} 


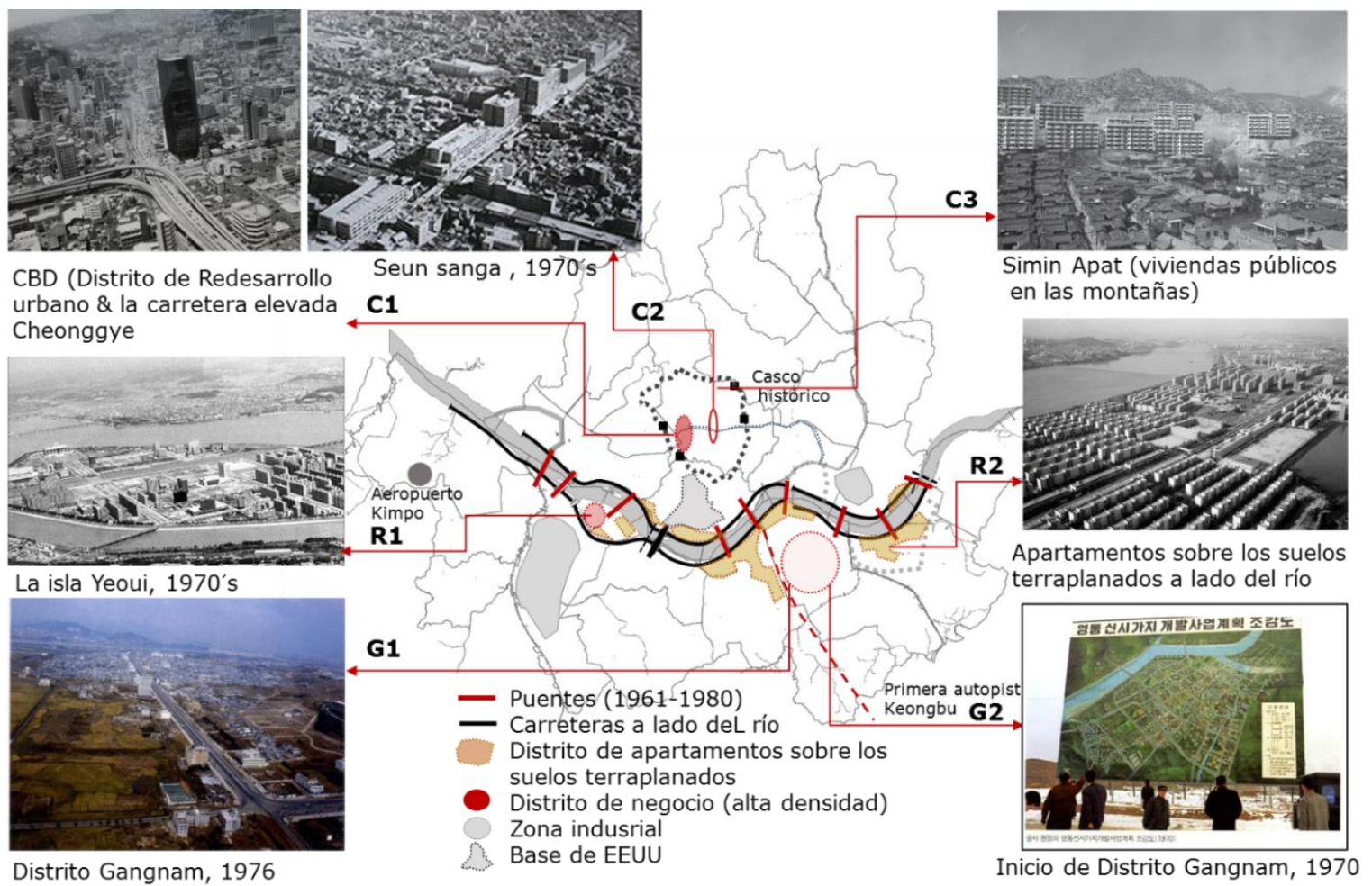

Figura 4: Grandes operaciones urbanísticas de seúl, 1960`s -1970`s, Fuente: Trabajo propio basado en “Look at Seoul through the Pictures $n^{\circ} 4,5$ ", Seoul Metro Politan Government $(2005,2008)$.

Mientras el Sur de la ciudad entraba en este proceso acelerado de desarrollo, en el centro histórico se acometía la construcción de numerosas infraestructuras como grandes vías y pasos elevados, desprovistos de escala humana, o el soterramiento de varios arroyos como el Ceonggyecheon (Ver C1 en Figura-4), transformados en infraestructuras de transporte. Con el nuevo proyecto de Redesarrollo Urbano impulsado en la época, distintos barrios vulnerables o afectados por las nuevas infraestructuras fueron realojados en proyectos puntuales de vivienda pública "Simin-Apat"4 (Ver C3 en Figura-4) en las colinas, pero en mayor medida comenzarían a ser obligados a migrar de forma masiva hacia la periferia Sur de la ciudad.

Además, el centro histórico comenzaría a ser la región preferida para el desarrollo de megaproyectos inmobiliarios, como el Complejo Seun-Sanga ${ }^{5}$ (Ver C2 en Figura-4), o la construcción del distrito hotelero internacional en los alrededores del Ayuntamiento de Seúl. Se delimitó el distrito de Redesarrollo urbano (1973), en torno al palacio Keongbok y Namdaemoon (Puerta del Sur), propiciando a construcción de enormes torres de oficinas (con una manifiesta voluntad de competir en altura, como muestra la prensa de la época), que tenían la voluntad y el soporte administrativo de ser la nueva imagen de modernidad en Seúl, promoviendo la entrada de capital empresarial y financiero internacional.

${ }^{4}$ Construyeron para los pobres en las montañas de Seúl (1969-1972): 32 de barrios, 447 edficios, 17,365 casas, $36 \mathrm{~m}^{2}$ de superficie por vivienda, casi fueron demolido después de un hundimento. http://opengov.seoul.go.kr/seoul/

5 Fue diseñado por el arquitecto KIM, Su-Geun, planeó con el concepto megaestructura de Danke Genzo, los ciudadanos se quejaban por sobre-escala. SOHN, J.- M. 2003, vol 2. 
Tanto el desenfreno inmobiliario del Sur del río, como las restricciones institucionales en el norte del río contribuyeron a sentar la base de un desarrollo desequilibrado. La gran escala y consiguiente homogeneidad de los proyectos residenciales, la desigual distribución de los nuevos equipamientos públicos, o la localización de grandes áreas de realojo de población vulnerable, conformaban un modelo de grandes áreas homogéneas y especializadas, dispersas en el territorio. Es interesante ejemplificar esta tendencia con la construcción de dos tercios de los puentes, construidos en esta época, hacia el sureste y los proyectos residenciales en auge, y sólo un tercio hacia al Oeste, las zonas industriales y de realojo.

\subsection{Herencia}

El perfil desordenado de Seúl, sin aparente orden tipológico, la coexistencia de grandes zonas homogéneas, atravesadas sin noción de escala humana por enormes infraestructuras, son el paisaje dominante en el Seúl actual, iniciados en esta época bajo el mito del desarrollo económico y la estrategia defensiva. La falta de recursos reales en materia de capital, tecnología, o herramientas de planeamiento urbanismo, generó un menor desarrollo cualitativo frente a un crecimiento cuantitativo, casi ornamental, en el que la administración centraba la atención en la apariencia del desarrollo, sin considerar conceptos como la calidad de vida de los ciudadanos o el medio ambiente, hasta la fecha absolutamente ajenos al discurso desarrollista imperante en esta etapa.

\section{Etapa II ( 1980-1992)}

En la década de 1980, la extensión hacia el sur del río continuó creciendo de manera vertiginosa. La celebración de los primeros eventos deportivos internacionales en Corea marcaban la enorme motivación inmobiliaria del momento.

\subsection{Principales acontecimientos en el contexto urbano de Seúl}

Un mes después del fallecimiento del dictador, un nuevo golpe militar marcaba el inicio del gobierno denominado como post-militar $\left(5^{\circ}-6^{\circ}\right.$ República de Corea) por dos presidentes aún provenientes del seno del ejército. Fue esta una época de gran contestación ciudadana y luchas de clase, que finalmente empujó al gobierno, en 1986, a comenzar la transición hacia la democracia. En esta etapa, el gran crecimiento demográfico (desde los 8.4 millones en 1980, hasta los 10 de 1990, similar a la que se registra actualmente) motivó en gran medida el mantenimiento de la dinámica de fuerte desarrollo inmobiliario.

\subsection{Estrategias urbanas estatales, y planes urbanísticos metropolitanos}

En esta etapa, los eventos urbanos de mayor importancia son las dos celebraciones deportivas acontecidas en la década, y la acelerada expansión de la conurbación difusa hacia el sur del río Han:

- 2 Plan de Desarrollo integral del Río Han (1982-1986),

- Promoción de construcción de barrios nuevos con motivo de los eventos deportivos (como el Complejo Deportivo Jamsil)

- Mercado inmobiliario promotor de grandes ciudades satélite de nueva planta y tipologías homogéneas de vivienda en altura, y valor económico similar.

\section{Planes urbanísticos de etapa II: Anexo-1}

- 1980: Long Term Schematic Plan and Mid-Term Plan for Seoul Urban Development toward the 2001 ,

- 1984: City Master Plan toward the 2000, 


\section{- 1990: City Master Plan toward the 2000}

El Plan de Seúl de 1980 planificó el desarrollo de la estructura poli-nuclear introduciendo conceptos como la proximidad al transporte público (destaquemos que en esta época se empezó a desarrollar la red de metro, y el concepto estaría ya presente en todos los planes posteriores como los de 1984 y 1990), la autosuficiencia administrativa de las regiones satélite (concepto muy presente en la planificación de 1981 a 1983), o la planificación sostenible y la optimización de recursos (introducida en el plan de 1984) El plan de 1990 sería el primer documento de carácter realmente legislativo, y ya incluía en sus contenidos conceptos vinculados al aumento de zonas verdes y la protección de zonas naturales.

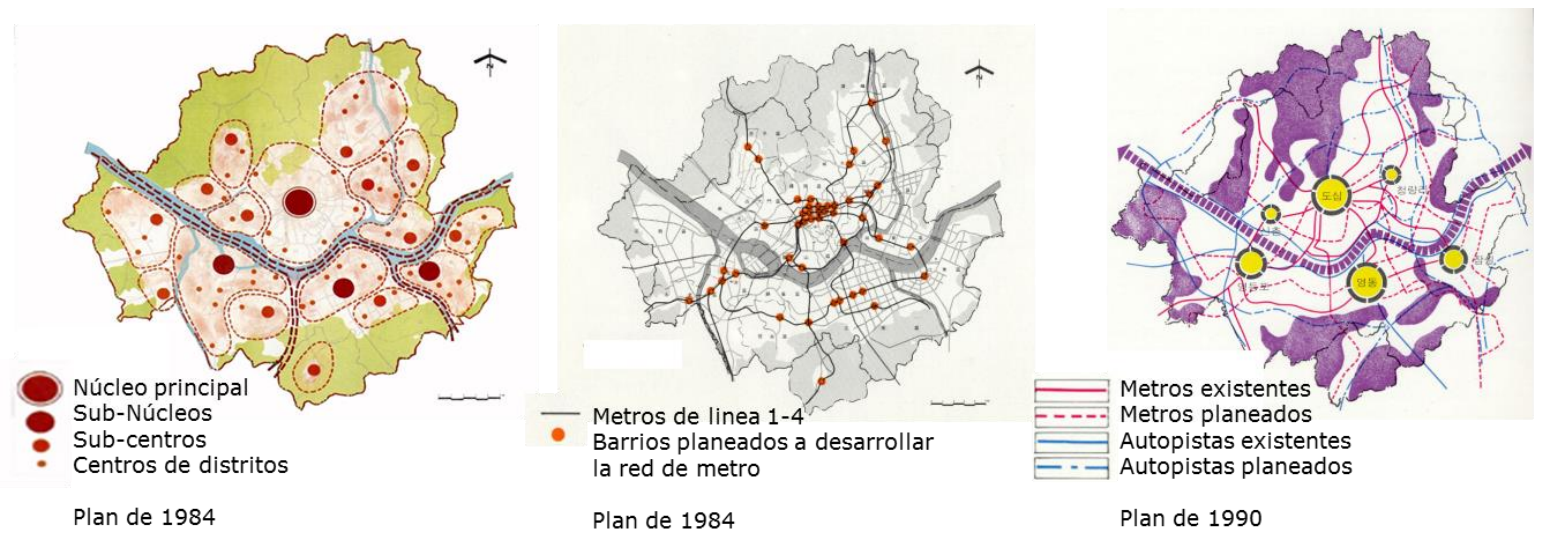

Figura 5. Cambio del plan de estructura espacial de Seúl: 1984, 1990 Fuente: Plan de Seúl, 1997.

\subsection{Grandes operaciones urbanísticas}

Para celebrar los dos eventos deportivos internacionales se propuso, en el $2^{\circ}$ Plan del desarrollo del Río Han, la ampliación y extensión de las autovías marginales al río hasta el aeropuerto Kimpo, cambiándose el nombre de la vía al sur del rio por Olympic Daero(Gran Calle de los Juegos Olímpicos). La infraestructura necesaria para los juegos motivó la construcción de grandes parques a largo del río, y una ambiciosa estación de depuración para recuperar el cauce, muy dañado por la construcción de diques en etapas anteriores. Aunque el acceso peatonal a la zona era muy complicado (una serie de túneles por debajo de numerosas vías de transporte), Seúl abría por primera vez grandes zonas de espacio público para el disfrute ciudadano (Ver R1 en Figura-6).

En el caso del distrito Jamsil (sur-este del río, Ver R2 en Figura-6), se promovió la construcción de un complejo deportivo integral, con un barrio para deportistas (el Distrito Jamsil, formado por grandes unidades de apartamentos en altura). En el sur-oeste del río (en la isla Yeoui, se erigieron diversos rascacielos con el beneplácito de la administración, (como el 63 Building, de 250m de altura, o la LG Twin Tower. Estas operaciones compartían la misma herencia de grandes zonas de desarrollo inmobiliario de monocultivo tipológico, y pese a introducir conceptos más avanzados en la planificación, seguían produciendo situaciones de transformación dramática del territorio de Seúl, como la del distrito Mokdong (Ver R4 en Figura-6), un polémico caso de desalojo de un barrio vulnerable para la construcción de grandes unidades de vivienda en altura para visitantes de los eventos deportivos. 


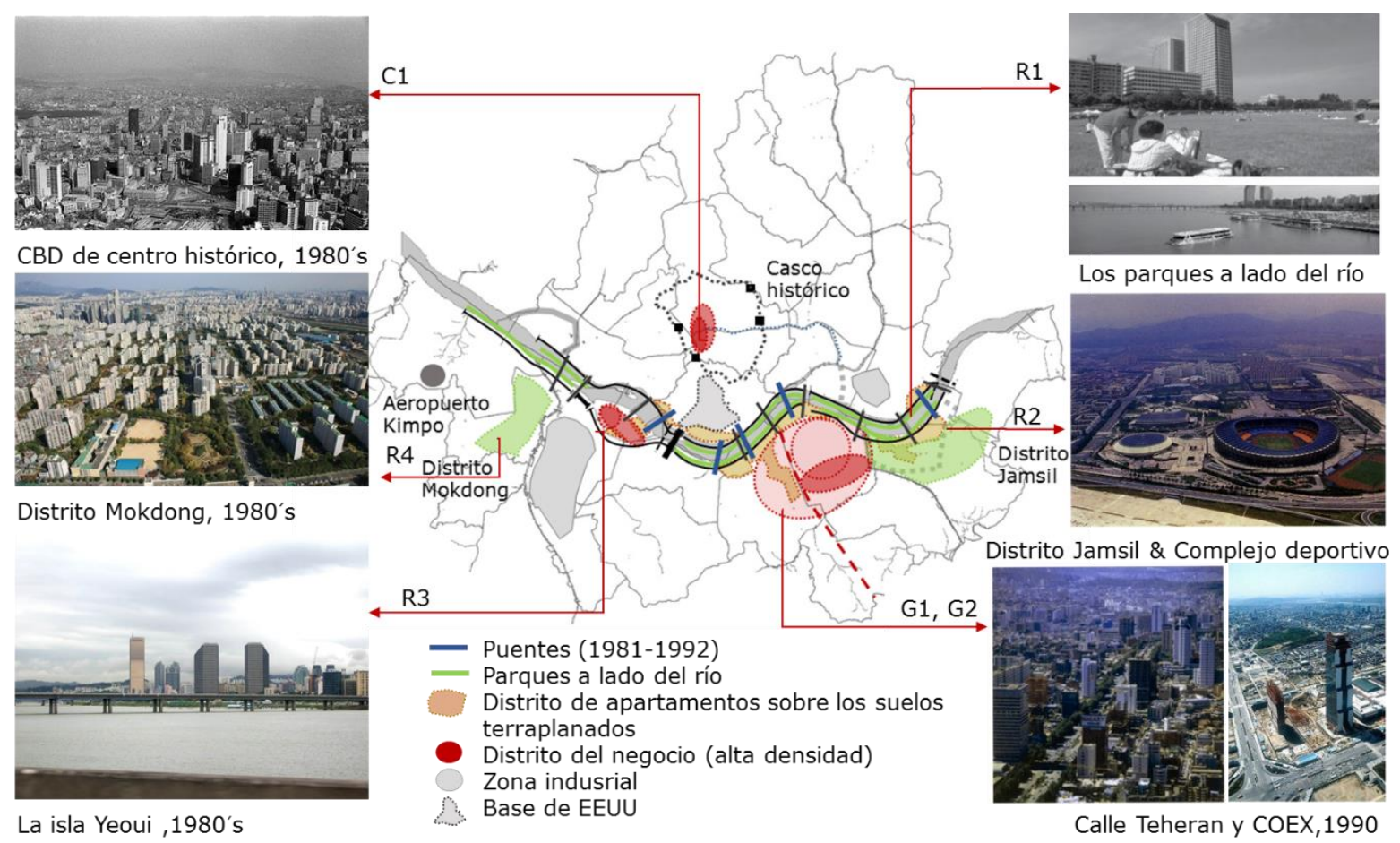

Figura 6: Grandes operaciones urbanísticas de seúl, 1980`s, Fuente: Trabajo propio, “Look at Seoul through the Pictures $n^{\circ} 6$ ", Seoul Metro Politan Government (2008).

En esta época, sin duda, el área con un desarrollo más paradigmático del momento es el Distrito Gangnam (sur-este del río), que atrajo hacia sí un porcentaje muy elevado de los edificios administrativos por el traslado de los equipamientos públicos (Fiscalía Judicial, COEX (Convention \& Exhibition / Asociación de comercio internacional de Corea, Ver G2 en Figura-6), Oficina de Administración Aduanera, Biblioteca Nacional y numerosos colegios, etc.). Además, la construcción en 1984 de la línea 2 del metro, que conectaría los principales equipamientos de la región (se sumarían las líneas 3 y 4 en 1985) y propició una extensión lineal de la zona en desarrollo en torno al metro (un buen ejemplo es la Calle Teherán, desde la estación Jamsil hasta el estación Gangnam, Ver G1 en Figura-6) con un tejido de construcciones en altura. Llegado este punto, la isla de Yeoui y el distrito de Gang-Nam habían conseguido la hegemonía en el ránking de actividad económica de la ciudad, siendo dos Núcleos de la llamada "Ciudad de 3 Núcleos".

Mientras, el centro histórico continuaba su deterioro. Sólo una pequeña parte del mismo, el área de "Re-desarrollo urbano" (principal Núcleo, Ver C1 en Figura-6), consiguió acometer mejoras urbanas, con la construcción de edificaciones de rascacielos y otros tipos de equipamientos públicos, así como pequeñas estaciones de autobús, aún privadas y exclusivas de zonas residenciales asociadas a población tradicional de renta muy elevada. El resto de la región fue transformando antiguos usos en declive, como los ancestrales mercados de materiales de construcción, ubicados tradicionalmente dentro de las cuatro Puertas antiguas que fueron desalojados por 'inadecuados', dejando grandes áreas del centro libres para la especulación imperante, y eliminando los restos del tejido complejo y compacto del núcleo antiguo de Seúl.

En éste proceso, el desequilibrio del desarrollo entre el norte y el sur del río se hizo cada vez más patente en las grandes diferencias de morfología urbana entre el centro antiguo cada vez más desmembrado, y las nuevas zonas de desarrollo homogéneo y de gran escala. Más puentes fueron añadidos a la red viaria de la ciudad, y de nuevo se observa una gran descompensación territorial 
en su disposición, con un solo puente hacia el Oeste (conectando la isla de Yeoui y su distrito financiero) y el resto hacia el Este (Gang-Nam)

Para el tejido residencial, fue esta una etapa de gran desarrollo inmobiliario, y de relativa experimentación tipológica, aunque segregada en el espacio. Es interesante comprobar la adopción del concepto y la palabra 'neighbourhood' para referirse a los nuevos distritos residenciales de la época, como Jamsil y Mokdong, que incluían una oferta de equipamientos educativos y zonas verdes, y eran muy valorados por la sociedad, lo que justificaba la creación de más ciudades satélite dada su aceptación en el mercado. Pese a la predominancia de tipologías verticales, fue ésta una etapa de cierta experimentación tipológica. Se actualizó el repertorio tipológico de vivienda colectiva con proyectos de baja densidad y altura (menos de cinco plantas), llamados Yeolip jutaek (manzanas compactas), Dasedai jutaek (edificios aislados), y Dandok jutaek (viviendas unifamiliares). No obstante, la implementación de grandes áreas de desarrollo inmobiliario sin nociones de su contexto previo, agravó su fragmentación, demasiado homogénea en los conjuntos construidos unitariamente, y demasiado heterogénea en la escala territorial.

\subsection{Herencia}

En conclusión ésta etapa reafirmó la tendencia de dispersión y especialización espacial, a través del desarrollo de grandes proyectos unitarios y una evidente voluntad de concentración de funciones, tecnología y capital, bien ejemplificados por los proyectos de los eventos deportivos internacionales. Pese a que la apertura de Corea hacia el exterior comenzó a introducir conceptos avanzados de desarrollo urbano, la fuerza del mercado inmobiliario y la aún limitada capacidad de implementación de políticas de planificación, contribuyeron a mantenimiento de la tendencia de polarización social y espacial norte sur en el territorio de Seúl.

\section{Etapa III (1993- Hasta la actualidad)}

\subsection{Estrategias urbanas estatales, y planes urbanísticos metropolitanos}

El crecimiento del mercado inmobiliario, alimentado por una exitosa industria de exportación, y exultante por la mejora de su imagen al exterior que suponían las celebraciones internacionales, forman el marco coyuntural de las últimas dos décadas. El cambio al gobierno democrático (1993) y la independencia total (1991), auspiciaron la aparición de las primeras instituciones de planificación urbana. Con la proyección de "Capital Seúl, 600 años (1994)", empezó a orientar a una ciudad global, el paradigma de la política urbana se ha transformado en la búsqueda del desarrollo sostenible y el equilibrio territorial, siempre bajo un marco conceptual de ciudad competente en el mercado global. Las administraciones locales han puesto sus esfuerzos en resolver los problemas que se han acumulado por la urbanización con gran velocidad de las dos etapas anteriores, mediante la aplicación de herramientas de regeneración urbana, recuperación y protección de zonas naturales y el equilibrio entre distintos centros urbanos.

\section{Planes urbanísticos de etapa III: Anexo-1}

- 1997: City Master Plan toward the 2011,

- 2009: City Master Plan toward the 2020

En el plan de 1997, se identifica la necesidad de reconstitución de la estructura espacial del territorio de Seúl y se propone la creación de sub-centros locales, y una zona de desarrollo estratégico en torno al río y los vectores de comunicación de las zonas satélite heredadas de épocas anteriores. De esta manera, se trata de reducir la escala del funcionamiento local y controlar la excesiva dispersión de los previsibles nuevos núcleos de urbanización. Y se propusieron 5 Distritos para desarrollo estratégico en torno del río. En el plan de 2009, denominado el "renacimiento del río Han", y los 3 núcleos anteriores con su estructura de sub centros definieron 5 áreas de desarrollo local según 4 direcciones de conexión de la ciudad 
existente en sí misma, y con el territorio circundante, además de establecerse dos grandes ejes ecológicos, uno este-oeste en paralelo al río Han, y uno norte sur de los montañas.

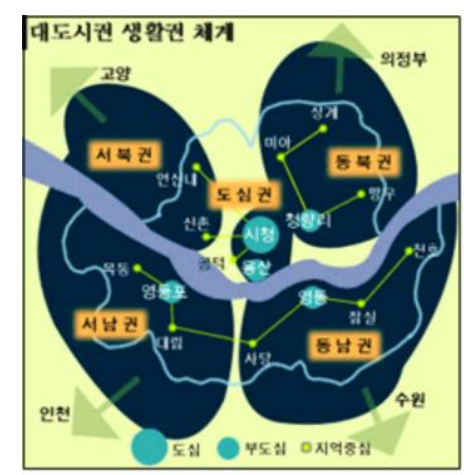

Plan de Estructura espacial, 1997

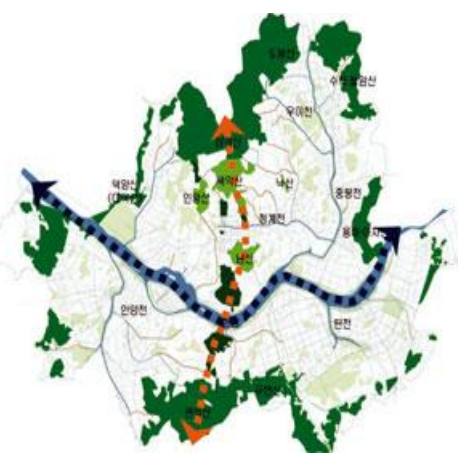

Dos ejes ecológicos, 2009

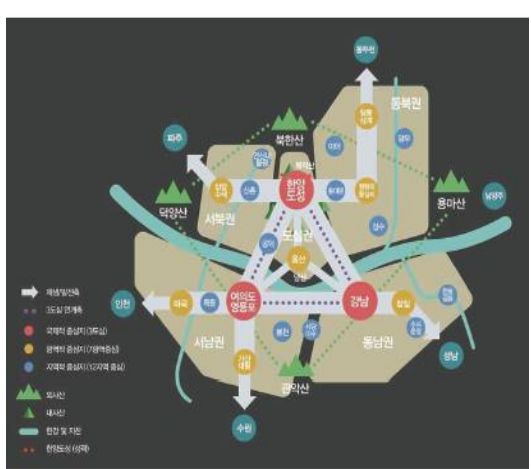

Plan de Estructura espacial, 2009

Figura 7. Cambio de estructura espacial de Seúl: 1997, 2009 Fuente: Plan de Seúl, 1997, 2009

\subsection{Grandes operaciones urbanísticas}

Los cinco distritos estratégicos propuestos en el plan de 1997, y las áreas de "renacimiento del río Han" en torno al río, se justificaron por la idea de la recuperación de la naturaleza de las islas y promoción del desarrollo en el oeste del río para el equilibrio territorial. Pese al intento por introducir estos nuevos conceptos de planificación, se observa que la nueva distribución espacial no ha abordado la cuestión tipológica del desarrollo.

Encontramos desarrollos de envergadura como la Sangam Digital Media City (un complejo promovido por la industria del digital media, Ver R1 en Figura -8), el Millenium Park (que está formado por cuatro parques: el 2002 World-Cup Park, el Parque Haneul y el Paque Noeul y el Parque Nanjicheon en la rehabilitada isla de Nanji que había sido convertida en un vertedero de material de demolición). Pese a establecerse estas áreas de crecimiento en el sector noroeste del río, promoviendo una distribución más equilibrada por el territorio, lo cierto es que se trata nuevamente de las grandes promociones homogéneas que aumentan la tendencia fragmentada de desarrollo en Seúl. Podemos observar otros ejemplos de gran escala como el Distrito Magok (complejo promovido la industria del conocimiento, cerca del aeropurto Kimpo), el Distrito Yongsan (distrito empresarial construido para la promoción del Negocio internacional), y el proyecto del Parque Yongsan (que ocupará el terreno vacío tras iniciarse el proceso de desactivación de la base militar de EEUU). Estos proyectos de gran escala con tipologías verticales de gran densidad, se vinculan a la idea predominante de la "ciudad competitiva", contrastando con los nuevos conceptos de equidad propuestos en los planes urbanos. 
Es interesante, no obstante, mencionar que por primera vez se comienzan a ejecutar otras actuaciones estratégicas de menor escala con una voluntad más integradora de un tejido complejo y con mayor conciencia del valor ambiental: la isla Tuk (Ver R2 en Figura -8) que cambió en suelo en la etapa I, se usó como campo de golf y paseos a caballo bajo el gobierno militar. Se convirtió en gran parque con diversos temas, competiendo en el ámbito de Regeneración urbana con los parques conocidos de las grandes ciudades. También en la isla Yeoui, la Plaza 516 (Ver R3 en Figura -8) que fue una gran plaza vacía para memorar el día de golpe militar, cambió por el parque natural, conectándose con los parques al lado del río. Y la ísla Seonyu fue usado por la planta de purificación de agua del río, según la ampliación de los sistemas existentes en otro lugar del norte del río (2001), se convocaron el concurso para reutilizar por un parque natural. En 2002, se abrió con buen diseño ${ }^{6}$ por la forma de reciclar los edificios y estructuras existentes, transformando en el parque ecológico (Ver R4 en Figura-8). Finalmente, casi todas islas del río Han, se transformaron en grandes parques con la intención de recuperación de la historia.

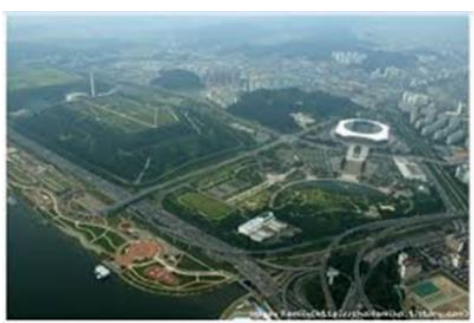

Distrito Sangam \& World-Cup Park

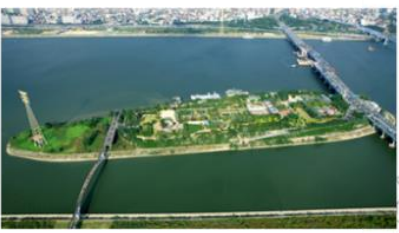

La isla Seonyu

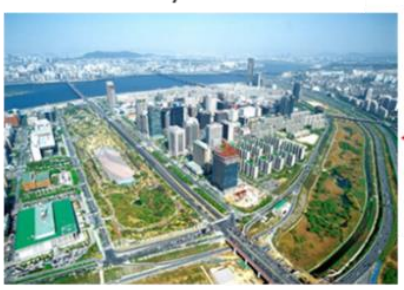

La isla Yeoui
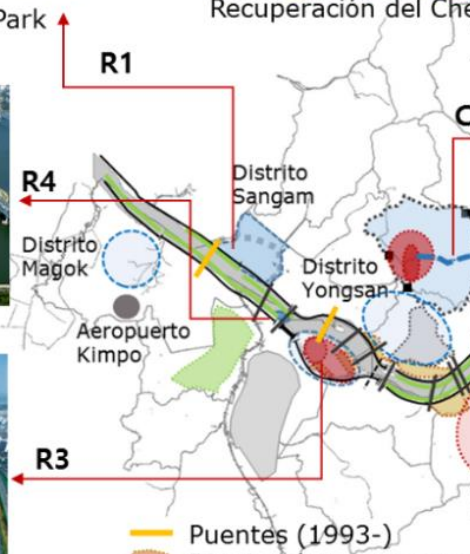

- Puentes (1993-) terraplanados

Distrito del negocio (alta densidad)

Zona indusrial

Base de EEUU

R1

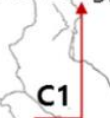

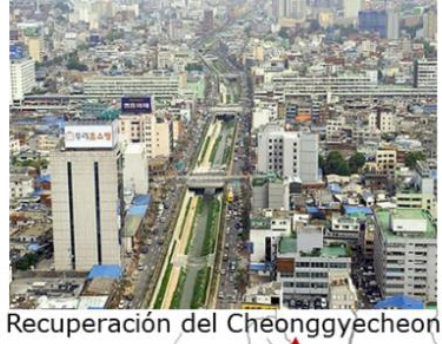
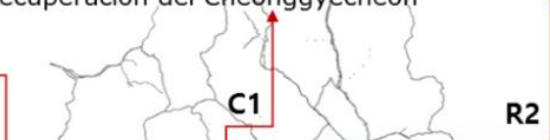

R2

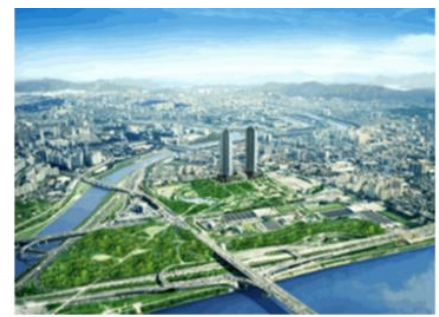

$\uparrow$ La isla Tuk (Ttukseom)

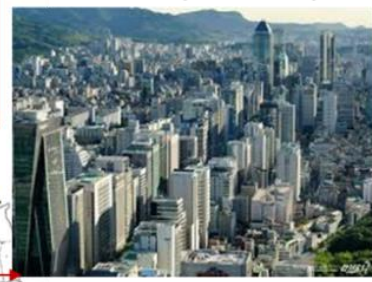

$\mathbf{G}$

Calle Teheran en Distrito Gangnam

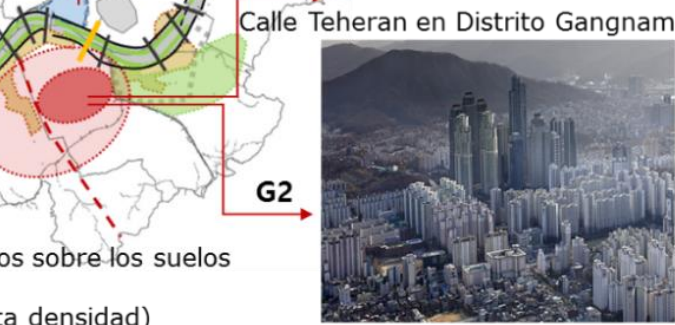

Tower Palace en Distrito Gangnam

Figura 8: Grandes operaciones urbanísticas de Seúl, 1990`s-, Fuente: Trabajo propio, http://parks.seoul.go.kr/, http://opengov.seoul.go.kr/seoul/

En el centro histórico, aunque aún se siguen construyendo nuevas tipologías de torre de gran escala en algunos puntos, se han acometido proyectos más sensibles al contexto de la ciudad antigua, como el proyecto de recuperación del arroyo "Cheonggyecheon (Ver C1 en Figura -8)", soterrado durante el régimen militar y descubierto ahora para valorizar la presencia histórica y ambiental del mismo; la restricción de nuevos desarrollos en el casco histórico, o la deconstrucción

6 Fue diseñado por CHEONG, Y.-S., and CHO, S.-Y. Se ganó el premio de 'AMERICAN SOCIETY OF LANDSCAPE ARQUITECTURE`' en 2004. CHEONG, Y.-S., and CHO, S.-Y. (2002). 
de carreteras y pasos elevados para mejorar el entorno peatonal y el ambiente urbano. Esta nueva valorización del centro provocó un rápido proceso de especulación inmobiliaria que, aún con la declaración de "Hanok Seoneon (2008) para conservar las casas tradicionales en el casco histórico, ha derivado en la gentrificación acelerada del mismo, desplazando cada vez más rápido a la diversa población tradicional.

En el sur del río, si cabe, se ha llegado a acentuar aún más la construcción de grandes promociones de tipología vertical en escenarios de altísima rentabilidad, cuya polarización ha sido manifiesta incluso en su jerarquización tipológica, como en el polémico caso del conjunto de Samsung Tower Palace ${ }^{7}$ (apartamentos en rascacielos con una fuerte jerarquización espacial en altura), una gran operación con el manifiesto fin de promocionar la potente marca electrónica (Ver G1, G2 en Figura -8).

El tejido residencial, la industria de la construcción ha sido motor de políticas de liberalización en la administración, permitiendo un gran margen de maniobra a los capitales privados en la urbanización de Seúl, hasta el punto de haber generalizado por completo la tipología de torre residencial como mejor apuesta para maximizar el beneficio sobre el suelo. Algunos autores expresan evidencias de una jerarquización de ésta tipología. En cuanto a su tipo, ubicación, marca de la empresa de construcción, tamaño del barrio, e incluso número de alturas. Se han llegado a producir demoliciones de zonas de torres residenciales de ya considerable envergadura, para ser sustituidas por torres aún más altas.

\subsection{Herencia}

Los elementos clave para entender ésta última ésta etapa son la aparición de la voluntad social por la regeneración urbana desde la recuperación de espacios y la calidad ambiental, hasta la reconstrucción de grandes zonas con cambios de uso para un mayor beneficio del conjunto. En general, el objeto de las operaciones institucionales ha sido recuperar valores ambientales e históricos en el contexto urbano que habían perdido en etapas anteriores, y paliar el desequilibrio entre los distintos centros de desarrollo. Pese a esto, la producción de espacio residencial (en su mayoría) ha agravado la fragmentación socio-espacial de Seúl, introduciendo nuevas manifestaciones de la misma como la jerarquización y competición entre distintas zonas de desarrollo.

\section{Conclusiones}

Hasta aquí, a través de las grandes operaciones urbanísticas en tres etapas distintas en torno al río Han, nos hemos aproximado a la evolución de Seúl.

En etapa I, como primera fase del desarrollo, la principal cuestión urbana fue la extensión hacia el sur para disminuir la fuerte centralidad heredada desde antes de la guerra, ampliando el área metropolitana al sur del río y trasladando equipamientos públicos a la misma, a la vez que se restringía el desarrollo al norte del río (exceptuando el pequeño distrito de Re-desarrollo en el centro histórico). La estrategia defensiva motivó la expansión hacia el sur del río iniciando un proceso especulativo afianzado por la fuerte inversión en las infraestructuras de la antigua zona agrícola, originando dese el comienzo una fuerte desigualdad de desarrollo norte-sur en la ciudad, que se evidenciaría en las primeras actuaciones inmobiliarias de gran envergadura en Gang-Nam (sur-oeste del río), la isla Yeoui (sur-oeste del río), y una pequeña parte del centro histórico (norte del río). El gran desarrollo en infraestructura (9 puentes sobre el Han y las autopistas en sus

\footnotetext{
7 Son 7 edificios de 42 a 93 plantas, se ejecutó Samsung en gran fricción con la comunidad de Seúl y con los habitantes de su alrededores (1999-2004).
} 
márgenes) fue ejecutado con una voluntad desconectada del valor natural y la memoria colectiva de sus habitantes (como el arroyo Cheonggye), sin mención de la escala humana totalmente perdida (infraestructuras de transporte en el centro urbano). La política migratoria forzada sobre la población más vulnerable, y la promoción del nuevo estilo de vida moderno sobre la población más acomodada, acentuó la dispersión hacia el sur de una manera segregada en inicio.

Es necesario entender que todos estos proyectos en la post-guerra inmediata, fueron ejecutados por una administración dictatorial y los alcaldes militares bajo el lema de la estrategia militar de dispersión y del desarrollo económico en una situación de carencia de recursos como capital, tecnología, o nociones de ordenación del territorio, y se centraron en el desarrollo aparente y cuantitativo antes que en la mejora cualitativa en la calidad de vida de los ciudadanos o el medio ambiente. No obstante, cabe destacar que este proceso se inició en un contexto de decisión unilateral desde las estructuras de poder, y sentaría las bases del modelo liberal posterior.

En la segunda etapa descrita, se llevó a cabo la extensión hacia el sur del río con gran cantidad de terreno urbanizado, con el leitmotiv del desarrollo ejemplificado en las dos celebraciones deportivas internacionales y el traslado de equipamientos públicos hacia nuevas áreas de desarrollo, y la construcción de gran cantidad de vivienda de alta densidad vertical. Más ejemplos de ésta fase se observan en la ampliación de la estructura viaria en el margen del río Han (5 puentes), con una distribución desigual este-oeste, la construcción de los gigantescos complejos deportivos, y la urbanización de Gang-Nam y Yeoui, que fueron a su vez produciendo problemas de deterioro en el núcleo antiguo, al abandonarse la actividad urbanística en el mismo, motivados doblemente por la nueva atracción de las zonas al sur del río, y una restricción impuesta a su desarrollo desde la administración local.

Pese a adoptar nuevas técnicas modernas de producción de espacio urbano y el crecimiento del capital y la tecnología en el país, la política urbana reforzaba el desequilibrio y la exclusividad entre el norte y el sur del río, entre los barrios nuevos y antiguos, con un proceso de edificación sin consideración del contexto urbano, que evidenciaban ya la gran limitación de la política planificadora a la sombra del incipiente mercado inmobiliario.

En la última etapa descrita, el paradigma urbano cambió por una voluntad mayor de regeneración urbana hacia el desarrollo sostenible y el equilibrio territorial, así como una mayor capacidad de planificación, auspiciadas por la llegada de la Democracia. Se observa cierta redistribución espacial en las zonas de nuevo desarrollo, así como proyectos puntuales de recuperación ambiental o urbana, pero la excesiva fragmentación, polarización y homogeneidad del espacio producido continúa siendo la tendencia importante, bajo la idea de la competitividad internacional de la ciudad, ahora totalmente inmersa en las redes transnacionales de transporte de personas y capital. Básicamente, ésta etapa ha adoptado una filosofía de crecimiento urbano más ordenado, incluso dentro de su fuerte ambiente de desregulación inmobiliaria, y con una mayor conciencia del contexto previo a las actuaciones, pero la cultura de la máxima optimización económica ha mantenido el patrón de crecimiento tipológico homogéneo y fuertemente jerarquizado.

En definitiva, el desequilibrio entre norte y sur del río en la ciudad de Seúl, primero motivado por una cuestión militar, y después acentuado por la rápida desregulación del suelo unida a políticas de migración forzada de población vulnerable, es un proceso altamente vinculado a una sociedad que ha conectado muy rápidamente su estructura socioeconómica a las grandes redes globales de intercambio, pero que también ha heredado un fuerte control estatal en su política territorial, primero totalmente centrada en el desarrollo económico, para poco a poco, y con la adopción de un sistema de gobierno democrático y las primeras políticas firmes de planificación, intentar equilibrar más su territorio en cuestiones de equidad social y ambiental. No obstante, pese a que las políticas de planificación han sido más presentes y conscientes del conjunto en la última etapa, la siempre creciente pujanza de los agentes económicos, ha agravado la tendencia a la segregación espacial que, si bien ha disminuido en escala territorial, se ha agravado en su jerarquización y polaridad social, evidente a niveles menores, como la escala de barrio y la tipología arquitectónica. 


\section{Referencias}

AHN, C.-M. (2013). “Bundanchejewa Seouleui dosigujo` (A Divided Korea and Urban Structure of Seoul under the Cold War)", Hyangto Seoul (Native Seoul), n 81, pp. 161-205.

AHN, C.-M. (2008). "Gaehangihueui Seouleui dosigujowa geonchukeui byeonhwa (The Changes of City Structure and Architecture of Seoul: Focused on the change of city borders and structure)", Dosiyeoksamoonhwa (History and Culture of City), $n^{\circ} 7$, pp. 59-101.

CHANG, K.-S. (2008). "Baljeongukgaeui gonggangaebal, 1960 1980nyeondae Seoul Hangangbyeon Apat jugeojiyeok hyeongseongwajeongeul jungsimeiro (The Spacial Development of Development State: The case of the Process of Residencial Area Fomation along the Han Riverside during the 1960 's and 1980 's in the Seoul, Korea)", Gonggangwa sahoi (Space and Society), n²5, pp. 194-210.

CHEON, G,-S. (2012), “1970nyeondae Park Chung-Hee Jeonggweoneui Gangnamgaebal (The Gangnam Development by the Park Chung-Hee Regime in the 1970's)", Yeoksamoonje yeongu (Study of history and cultura), $n^{\circ} 28$, pp. 9-37.

CHEONG, I.-H. (1996). "Yeouido dosigyehoike gwanhan yeongu: Kim Su-Geunui 1969nyeon gyehoikaneul jungsimeuro (A Study on the Urban Development of Yoido: Focused on the plan of Kim Su-Geun)", Journal of Institute of architecture, $\mathrm{n}^{\circ} 12$ (2), pp. 123-135.

CHEONG, Y.-S., and CHO, S.-Y. (2002). "Seonyudo gongwon (1) (Seonyudo Park (1)", Journal of the Institute of Culture of Architecture, $\mathrm{n}^{\circ} 254$ (2002-07), pp. 33-51.

KIM, E.-R. and LEE, M.-H (2003). “Seoulsi GangNam-Buk bulgyunhyeong yoine gwanhan yeongu ( A study about the factors influenced the disparity of the Southern and Northern part of Seoul)", Journal of the Institute of Urban Planning and the Territory of Korea, Conference octubre de 2003, pp. 289-298.

KIM, K.-J. (2012). "The study of urban form in South Korea”, International Seminar on Urban Form, 2012 ISSN 1027-4278, pp. 149-164 (158).

KIM, J.-H. and KIM, K.-H. (2010). "1974nyeon "Jamsiljigu jonghabgaebalgibongyehoik"eui seonggyeokgwa dosigyehoijeok uimi (A Comprensive Plan of Jamsil District in 1974: Its Implications and Characteristics for Future Urban Planning)", Institute of Urban Planning of Korea, $n^{\circ} 11(4)$, pp. 41-57.

KIM, S.-H. (2013). "Changes in Urban Planning Policies and Urban Morphologies in Seoul, 1960s to 2000", Architectural research septiembre de 2013; n¹5(3), pp. 133-141.

LEE, D.-H. and AHN, C.-M. (2011). "Seunsangae daehan dosi-geonchukjeok jaehaeseok (A Re-interpretation Urban-Architectural about Seunsanga)", Journal of the Institute of the History of architecture of Korea, Congreso Noviembre de 2011, pp. 355-367.

LEE, K.-S. and NOH, H.-B. (1994). Jidoroboneun Seoul (Look at Seoul through Maps), Seoul: Seoul Development Institute.

LEE, I. -G. and SEOUL METROPOLITAN GOVERNMENT (2006). "Cheong Gye Cheon Restoration Project: a revolution in Seoul", Actas del Congreso ICLEI 2006 disponible en http://worldcongress2006.iclei.org/uploads/media/K_LEEInKeun_Seoul_-_River_Project.pdf, consultado en Junio de 2015.

PARK, H.-C., LIM, H.-J., LEE, S.-C., MIN, H.-S. and YEO, H.-J. (2009), “Seoul eui doshi hyeongtae yeongu (Urban form study of Seoul)", Seoul Development Institute, Seoul.

SEOUL METROPOLITAN GOVERNMENT. Urban Planning of Seoul: 1966, 1978, 1984, 1990, 1997, 2009.

- (2005). “Sajineuroboneun Seoul 4: Dasi ileoseoneun Seoul (1961-1970) (Look at Seoul through the Pictures 4: Raise up again Seoul (1961-1970))", Institute of the publications of the History of Seoul, $n^{\circ} 4$. 
Seúl y el Río Han: 1945 - 2014

Territorios en Formación | 2015 | N09 | TRABAJOS FIN DE MÁSTER - Estudios Urbanos

- (2008). “Sajineuroboneun Seoul 5: Paengchangeul geodeubjaneun Seoul (1971-1980) (Look at Seoul through the Pictures 5: Continue to spread Seoul (1971-1980))", Institute of the publications of the History of Seoul, $n^{\circ} 5$.

- (2008). "Sajineuroboneun Seoul 6: Segyereul hyanghae peodeonaganeun Seoul (1981-1990) (Look at Seoul through the Pictures 6: Extend to the world, Seoul (1981-1990))", Institute of the publications of the History of Seoul, $n^{\circ} 5$.

SOHN, J.-M. (2003), “Seoul dosigyehok iyagi $1^{\circ}-5^{\circ}$ (The history of the Urban Planning of Seoul vol 1-5)". Seoul.

YOUN, E.-J. and JEONG, I.-J. (2009). “Gangnameui dosigonggan hyeongseonggwa 1960n yeondae dosigyehoik sanghwang yeongu (A study of the Formation of the Urban Space of Gangnam Area and the Urban Discourse in the 1960 's)", Journal of the Architectural Institute of Korea, $n^{\circ}$ 25(5), pp. 231-238. 


\section{ANEX0-1. Planos de usos del suelo}

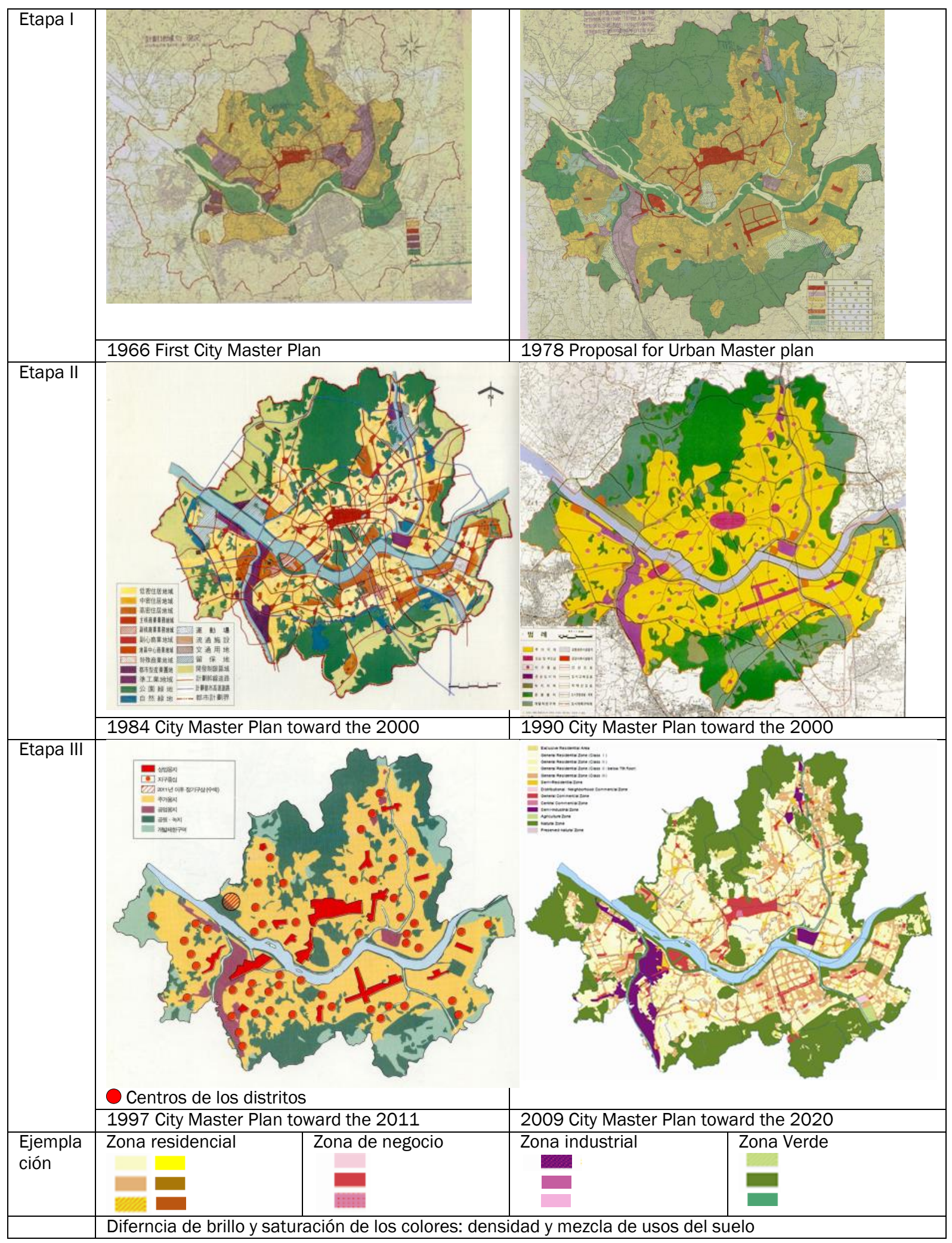

